\title{
Research Paper \\ The Role of Spontaneous Mind-Wandering and Dispositional Mindfulness in Predicting Obsession Symptoms in the College Students
}

\author{
Majid Mahmood Aliloo ${ }^{1} \mathbb{0}$, Abbas Bakhshipour $^{1},{ }^{*}$ Bahman Matinpour $^{2}$
}

1. PhD, Professor, Department of Psychology, Faculty of Education and Psychology, University of Tabriz, Tabriz, Iran.

2. MSc. Student, Department of Psychology, Faculty of Education and Psychology, University of Tabriz, Tabriz, Iran.

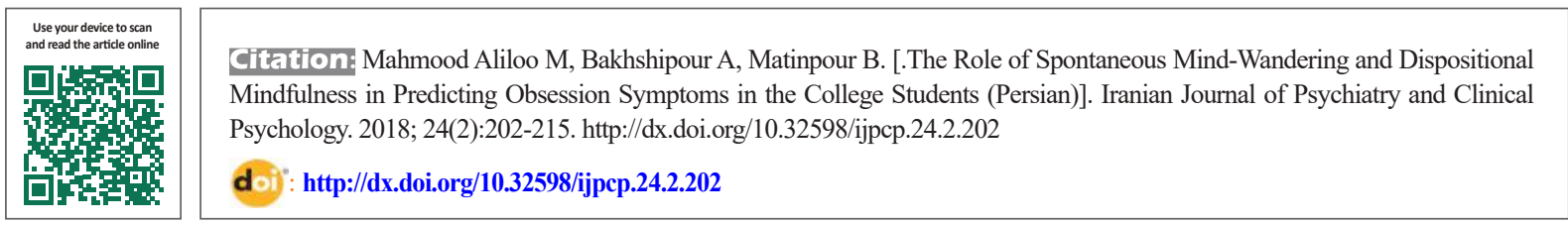

Received: 04 Sep 2017 Accepted: 13 Mar 2018

Keywords:

Obsession symptoms, Spontaneous and deliberate mind-wandering, Dispositional mindfulness

\section{A B S TRACT}

Objectives The aim of this study was to investigate the role of spontaneous or deliberate mind-wandering and dispositional mindfulness in predicting the obsession symptoms in college students.

Methods In a descriptive, correlated and tentative research, 391 students of Tabriz University were chosen and included in the inquiry via the available sampling method. The data were gathered using the Maudsley Obsessive-Compulsive Inventory (MOCI), Mind Wandering Spontaneous (MW-S), Mind Wandering Deliberate (MW-D) self-report scales and Five Facet Mindfulness Questionnaire (FFMQ). Pearson correlation-coefficient method and multiple regression were used to analyze the data simultaneously. Results Correlation results showed that the deliberate mind wandering $(r=0.19, P=0.05)$ and spontaneous mind wandering ( $r=0.64, \mathrm{P}=0.01$ ) have positive and direct relationship with obsession symptoms, while the components of mindfulness, the components of observation $(r=0.11, P=0.05$ sacting with awareness ( $r=-0.41, P=0.01)$, disrespect to inner experience $(r=-0.50, P=0.01)$ and lack of reaction to inner experience( $r=-0.61, P=0.01)$ have a negative and reverse relationship with obsession. The results from the concurrent regression analysis shows that from mind wandering components only spontaneous mind wandering variable $(P<0.01, \beta=0.51$ )and from mindfulness components, only act with awareness, non-judgmental orientation to one's present experience and non-reactive orientation to one's present experience $(\beta=-0.20, \beta=-0.22, \beta=-0.41)$ can predict obsession symptoms.

Conclusion The findings of this research emphasized the role of spontaneous mind wandering and components of acting with awareness, non-judgmental orientation to one's present experience and non-reactive orientation to one's present experience in predicting obsession symptoms in the college students. These findings will be beneficial in explaining obsession symptoms and their etiology in the college students.

\section{Extended Abstract}

\section{Introduction}

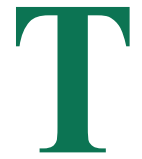

he aim of this study was to investigate the role of spontaneous and deliberate mindwandering and dispositional mindfulness in predicting obsession symptoms in the college students. Spontaneous and deliberate mind-wandering are types of intrusive thoughts, emphasized in recent researches. Spontaneous mind wandering is defined as thoughts that are irrelevant to the central task or thought. In fact, this type of mind wandering occurs automatically and without full consciousness of the person or he/she does not have con-

* Corresponding Author:

Bahman Matinpour, MSc. Student

Address: Department of Psychology, Faculty of Education and Psychology, University of Tabriz, Tabriz, Iran.

Tel: +98 (919) 4181634

E-mail: matinbahman@yahoo.com 
trol over his/her thoughts. This feature probably resembles the obsessive thoughts seen in OCD patients. Obsessive thoughts and spontaneous mind wandering both are considered as intrusive thoughts and share the features of repeatability and intrusiveness. Mindfulness has been defined as bringing one's attention to experiences occurring in the present moment. The Cognitive Assessment Model was introduced which emphasized on negative reaction upon inner experiences of OCD patients and noted that mindfulness might be beneficial in reducing obsession.

\section{Method}

Regarding the data gathering method, this study is a descriptive (non experimental) correlational design. In the present study, 391 Bachelors of Arts (B.A) students from Tabriz University who scored below 11 (lower than clinically significant scores) in Maudsley Obsessive-Compulsive Inventory (MOCI) were chosen using Morgan formula. The age of the participants was between 18-26 years. The available sampling method was used, and the data were gathered using the Maudsley Obsessive-Compulsive Inventory (MOCI), Mind Wandering Spontaneous (MWS), Mind Wandering Deliberate (MW-D) self-report scales, and Five Facet Mindfulness Questionnaire (FFMQ). Pearson correlation-coefficient method and simultaneous multiple regression were used to analyze the data. Data were analyzed using the IBM's SPSS 21 software package.

\section{Results}

In total, 391 subjects participated in the study. The mean and standard deviation of the participants were 50.1 and 32.22 , respectively. Correlation results showed that deliberate mind wandering $(\mathrm{r}=0.19, \mathrm{P}=0.05)$ and spontaneous mind wandering $(\mathrm{r}=0.64, \mathrm{P}=0.01)$ had a positive and direct relationship with obsession symptoms. Among the components of mindfulness, the components of observation $(\mathrm{r}=0.11, \mathrm{P}=0.05)$ ، acting with awareness $(\mathrm{r}=-0.41$, $\mathrm{P}=0.01)$, disrespect to inner experience $(\mathrm{r}=-0.50, \mathrm{P}=0.01)$ and lack of reaction to inner experience $(r=-0.61, \mathrm{P}=0.01)$ had a negative and reverse relationship with obsession. The regression coefficients showed that from mind wandering variables, only spontaneous mind wandering could predict obsession symptoms. The predictive coefficient of this variable is 0.51 , which shows its influence $(\mathrm{P}<0.01, \beta=0.51)$. Also, from mindfulness components, act with awareness, non-judgmental orientation to one's present experience and non-reactive orientation to one's present experience ( $\beta=$ $0.20, \beta=-0.22, \beta=-0.41$ ) could predict obsession symptoms. Also, deliberate and spontaneous mind wandering variables and components of dispositional mindfulness could explain 0.54 of variance of the obsession symptoms.

\section{Discussion}

The findings indicated that spontaneous mind wandering and components of act with awareness, non-judgmental orientation to one's present experience and non-reactive orientation to one's present experience could predict obsession symptoms. A shared conceptualization of spontaneous mind wandering and obsessive thought is that they happen when people experience undesirable failure in executive functions. Such experiences could make irrelevant thoughts enter the consciousness. Failure in executive functions might not only be linked to intrusive thoughts related to OCD, but it could also play a role in spontaneous mind wandering. Another finding was that components of act with awareness, non-judgmental orientation to one's present experience and non-reactive orientation to one's present experience could predict obsessive symptoms in a reverse manner. Didona stated that many psychological characteristics of OCD patients result from mindfulness deficits. For example, constant obsessions and compulsions are the opposite points of living mindfully in which the individual pays attention to them without judging whether they are right or wrong. Instead of being mindful and curious about their thoughts and actions, OCD patients feel guilty about their intrusive and unpleasant thoughts and their reaction to these thoughts results from their interpretation of them.

\section{Ethical Considerations}

\section{Compliance with ethical guideline}

The students participated in the study with full satisfaction and signed the informed consent, including the right to participate in or not to participate in research, privacy, and access to the results.

\section{Funding}

The present paper was extracted from the third author MSc. thesis and was financially supprted by University of Tabriz.

\section{Conflict of interest}

The authors declared no conflict of interest.

\section{Acknowledgements}

Hereby, we sincerely appreciate all the professors and students who helped us with this research. 


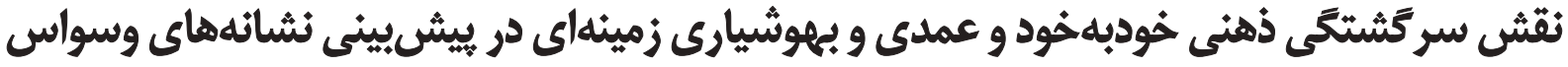 دانشجويان
}

\author{
مجيد محمودعليلو'، عباس بخشىيور' '"بهمن متينيور'
}

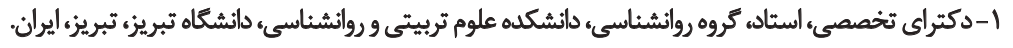

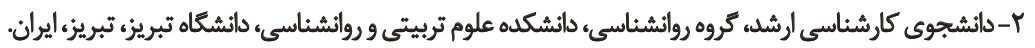

\begin{abstract}
حكيد
الهداف هدف يثروهش حاضر بررسى نقش سركشتكى ذهنى خودبهخود وعمدى و بهوشيارى زمينهاى در بيشبينى ثشائهاى وسواس دانشجويان بود.

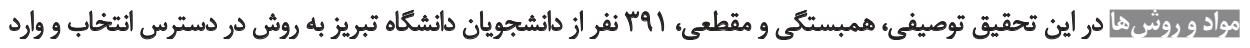

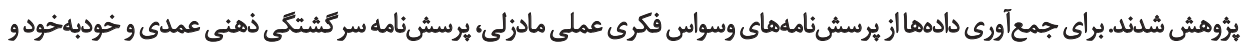

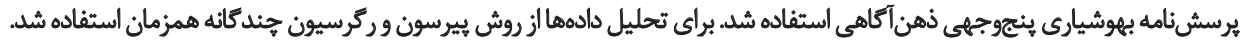

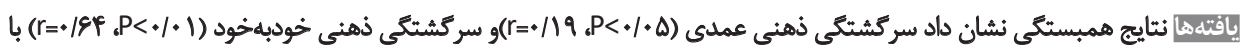

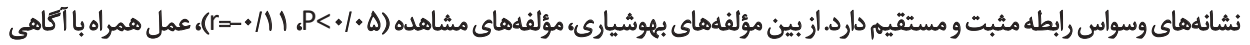

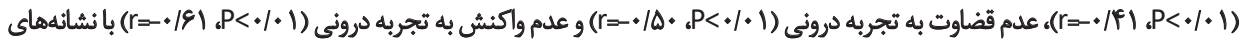

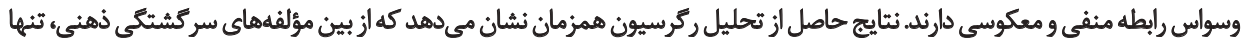

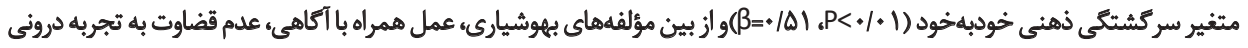

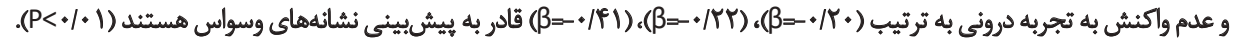

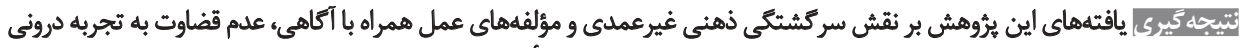

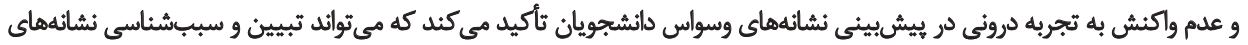

تاريخ دريافت: "Iا شهريور

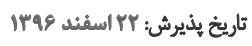

كليدواروها:

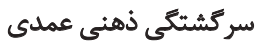

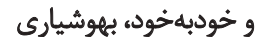

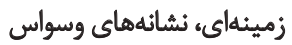

كاهي اوقات بسيارى از افراد افكار ناخواندهاي دارند و بعضي

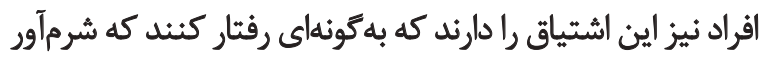

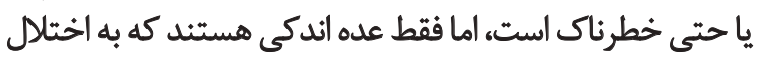

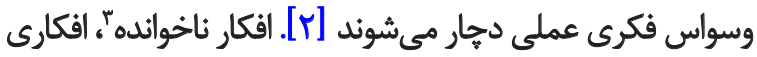

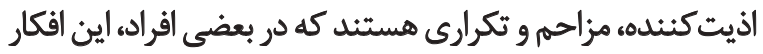

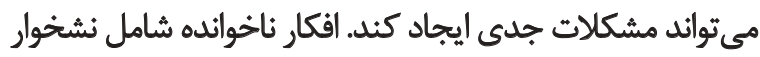

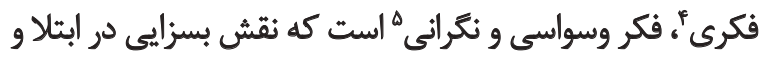
تداوم اختلالهاى خلقى، اضطر ابى (وسواس و اضطراب فرابي فراكير) و

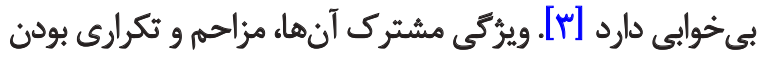
اين افكار است.
3. Intrusive thought

4. Rumination

5. Worry dalê

نسخه ينجم راهنماى آمارى و تشخيصى اختلالات روانى'

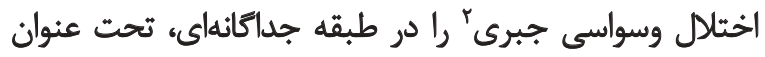

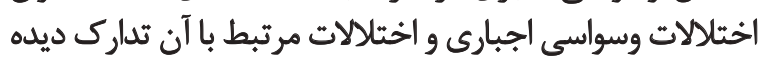

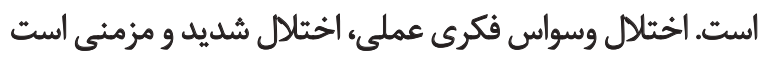

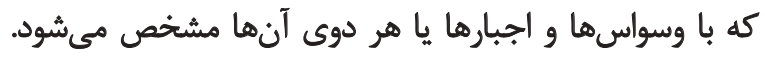

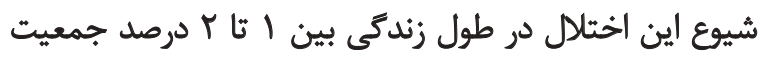

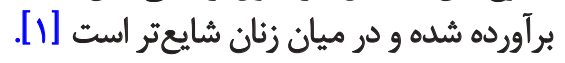

1. Diagnostic and Statistical Manual of Mental Disorders, Fifth Edition (DSM-5)

2. Obsessive-compulsive disorder 


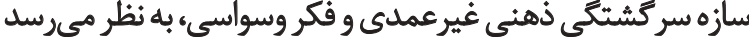

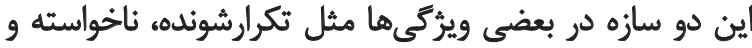

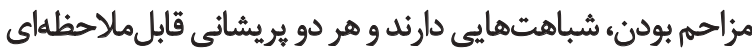

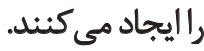

يكى از مؤلفههاى ديكّى كه در اختلال وسواسى جبرى در

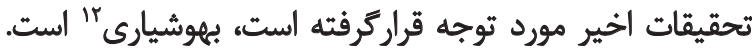

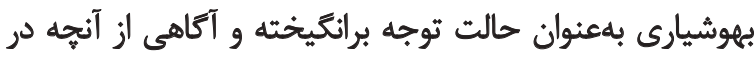

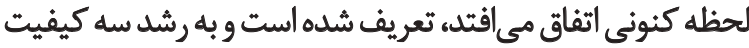

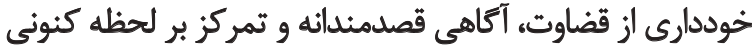

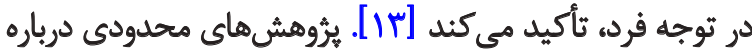
بهوشيارى زمينهاى در اختلال وسواسى جبرى انجام شده است.

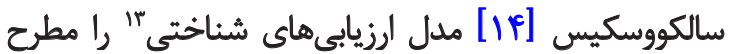

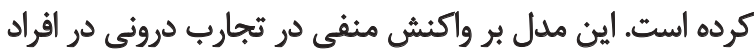

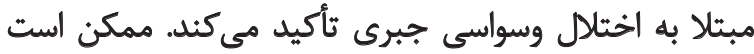

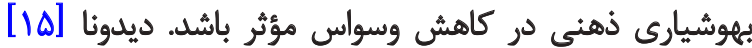

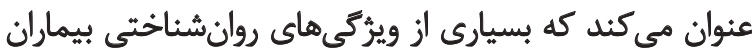

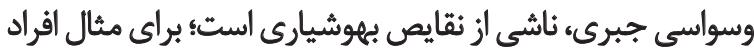

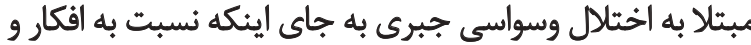

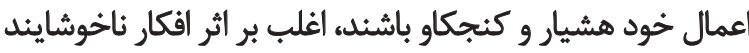

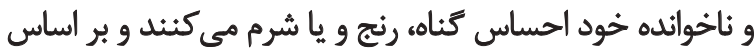

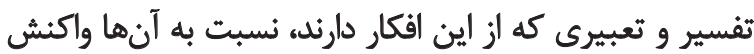

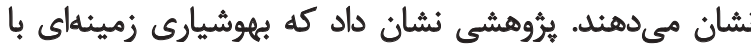

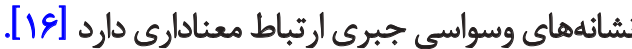
طى :ند دها قبل، مطالعات زيادى براى شناسايى عوامل

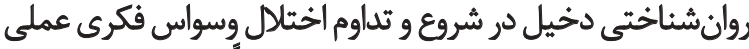

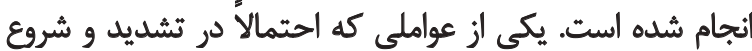

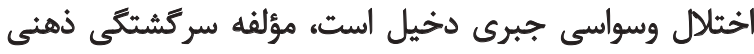

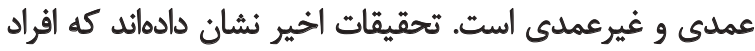

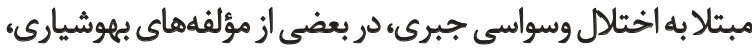

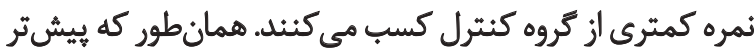

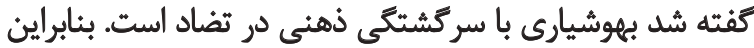

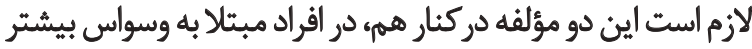

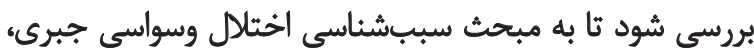

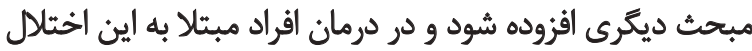

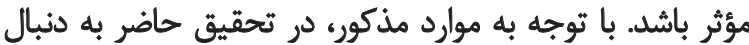

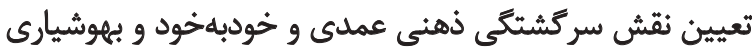

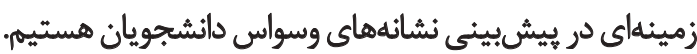

$$
\text { روشڤ }
$$

يُروهش حاضر با توجه به نحوه كردآورى دادهها، جزء تحقيقات

12. Mind fullness

13. Cognitive Assessment Model

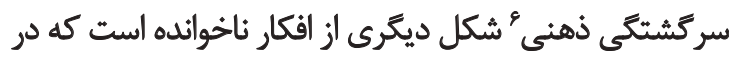

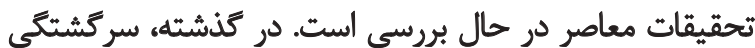

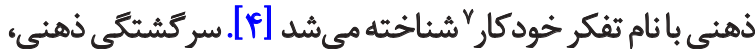

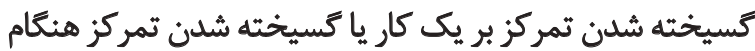

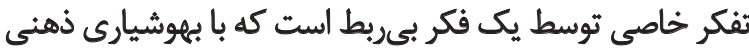

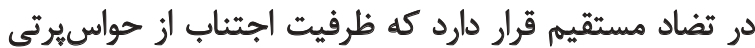

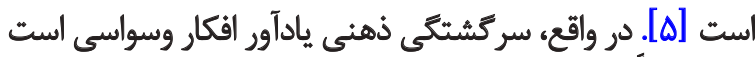

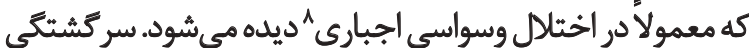

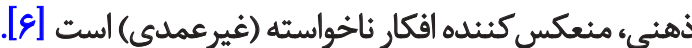

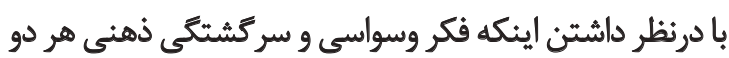

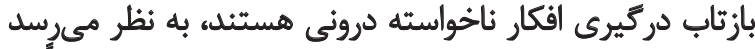

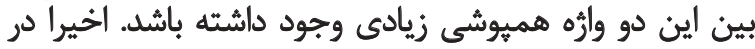

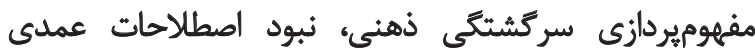

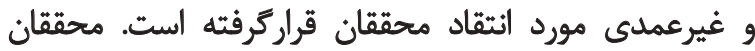

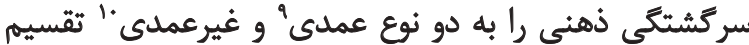

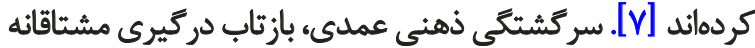

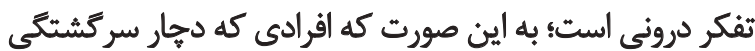

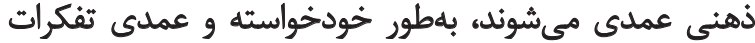

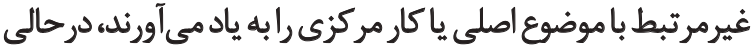

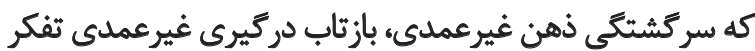

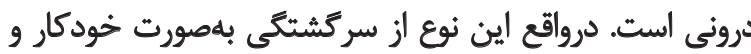

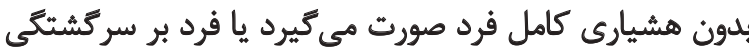

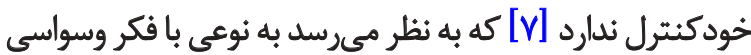
هميوشى دارد.

در ئروهشى سلى و همكاران [م] نشان دادند كه بين

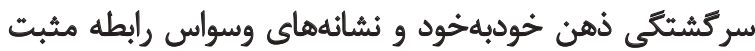

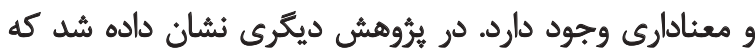

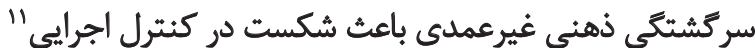

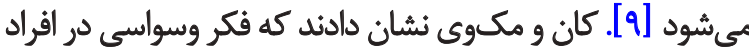

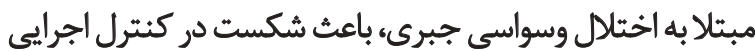

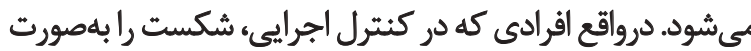

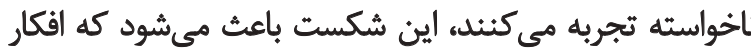

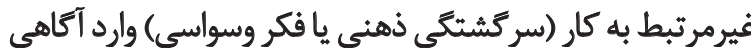

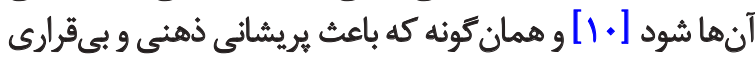

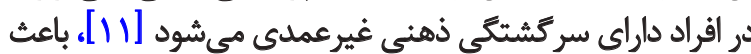

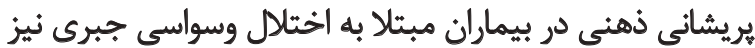

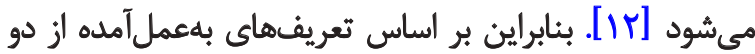

6. Mind wandering

7. Auto thinking

8. Obsessive-Compulsive Disorder (OCD)

9. Mind wandering deliberate

10. Mind wandering spontaneous

11. Failure of executive control 


$$
\text { ضريب آلفاى كرونباخ Af|Af • بود. }
$$

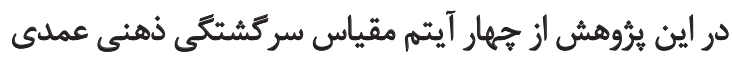

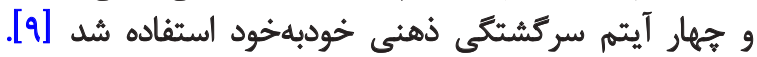

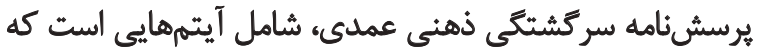

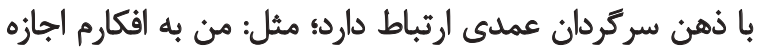

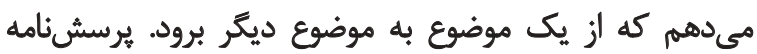

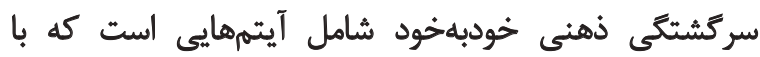

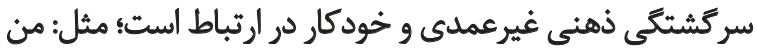

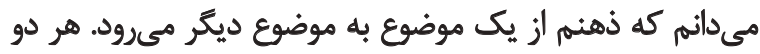

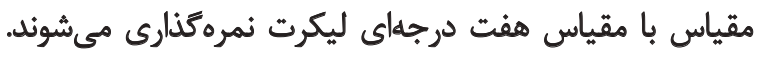

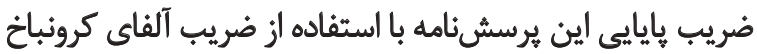

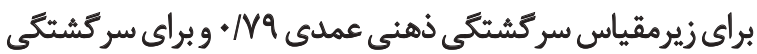

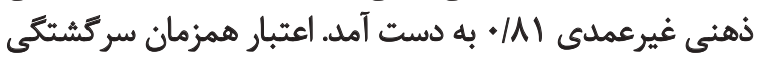

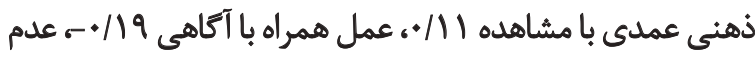

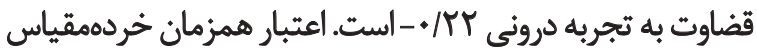

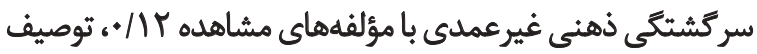

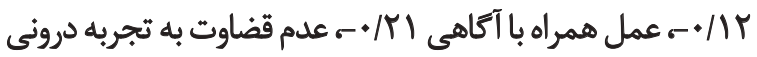

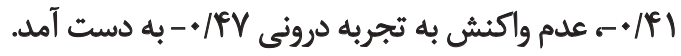

$$
\text { يوسش نامه وسواس فكرى عملى مادزلي }
$$

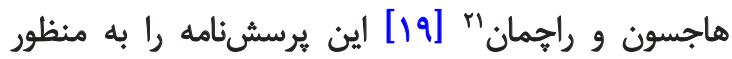

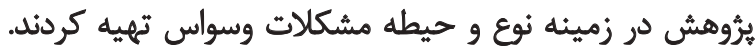

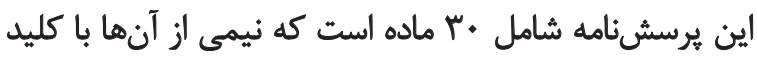

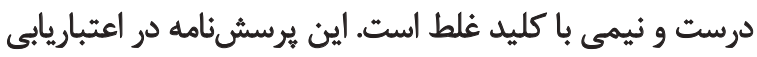

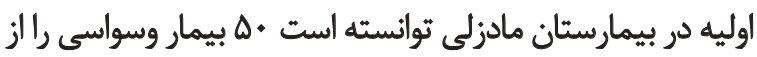

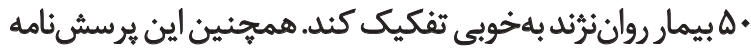

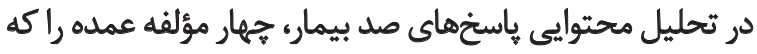

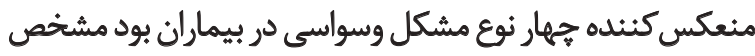

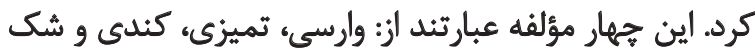

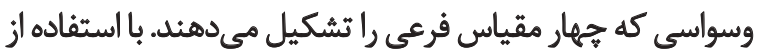

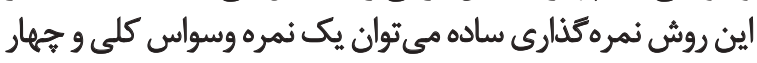

$$
\text { نمره فرعى به دست آورد. }
$$

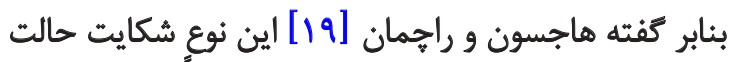

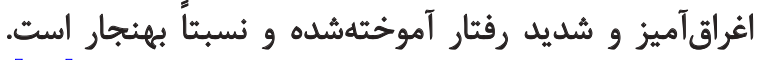

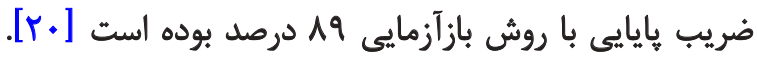

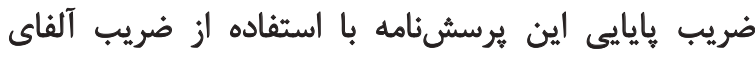

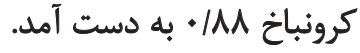

19. Mind Wandering: Deliberate (MW-D)

20. Mind Wandering: Spontaneous (MWS)

21. Hodgson \& Rachman

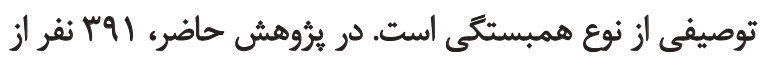

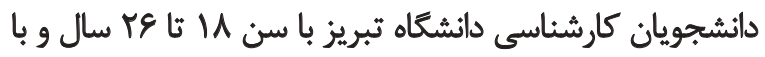

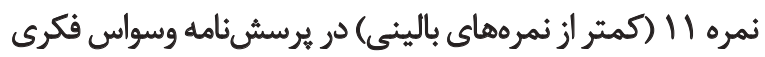

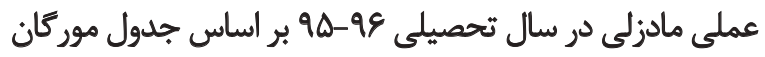

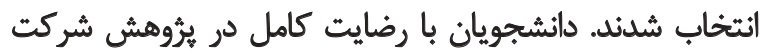

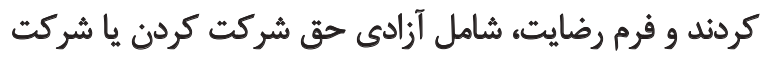

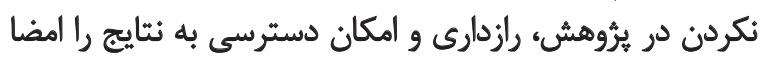

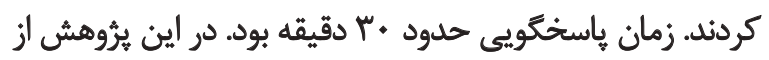

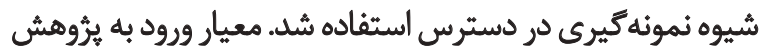

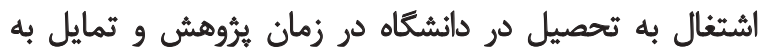

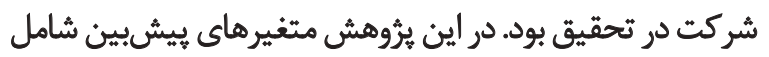

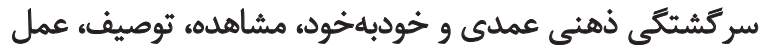

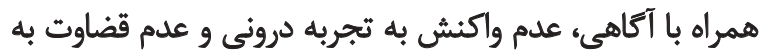

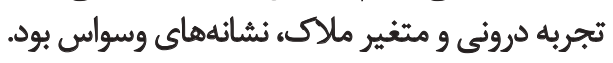

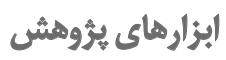

برسش نامه بهوشيارى نينجوجهى 18

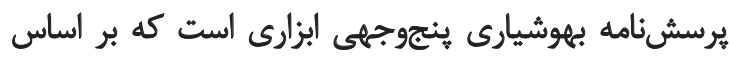

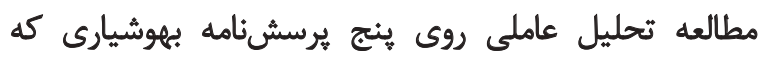

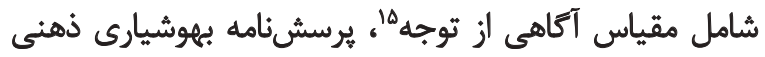

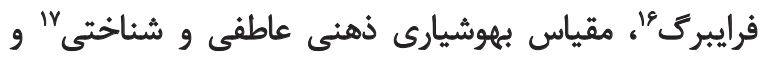

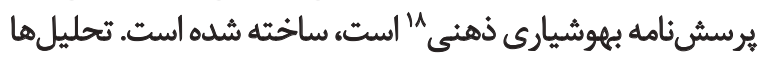

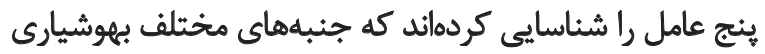

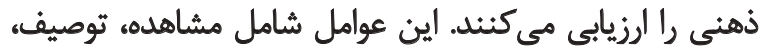

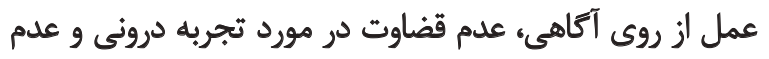

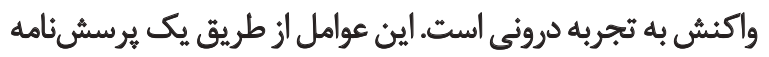

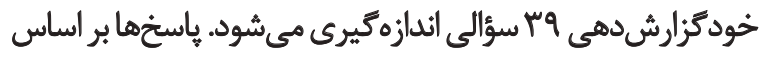

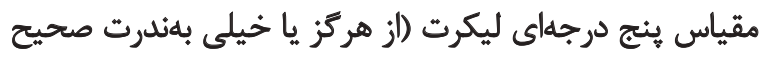

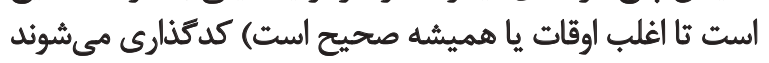

[IV]

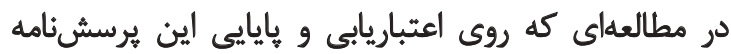

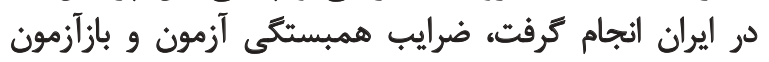

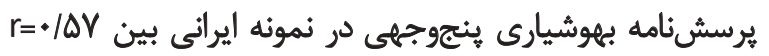

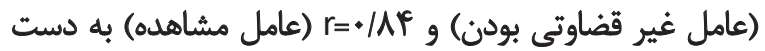

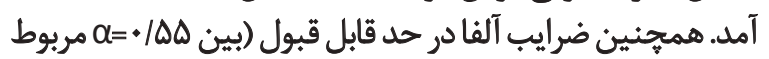

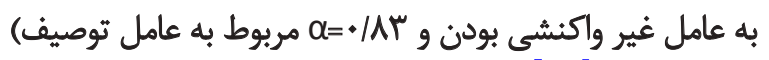

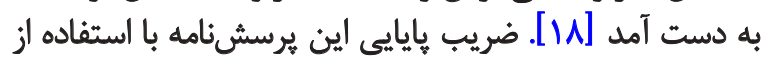

14. Five Facet Mindfulness Questionnaire (FFMQ)

15. Mindfull Attention Awareness Scale (MAAS)

16. Freiburg Mindfulness Inventory (FMI)

17. Cognitive and Affective Mindfulness Scale (CAMS)

18. Mindfulness Questionnaire (MQ) 


\section{ييشفرض استقلال مشاهدات}

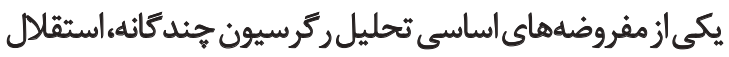

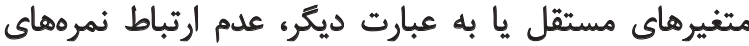

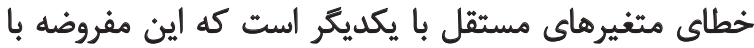

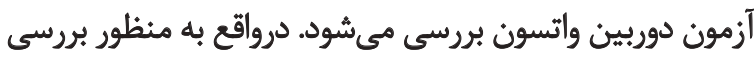

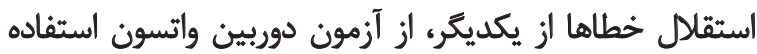

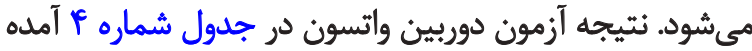

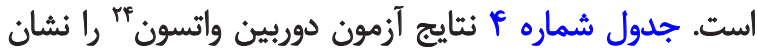

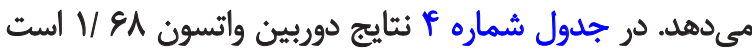

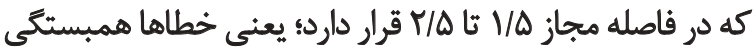
ندارند و مى توان از ركرسيون استفاده كرد.

$$
\text { يبش برض همخطى مثغير هاى يُوهش }
$$

همخطى هب بين متغيرهاى مستقل وضعيتى است كه نشان

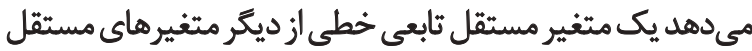

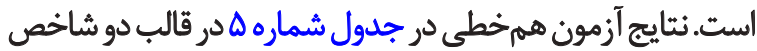

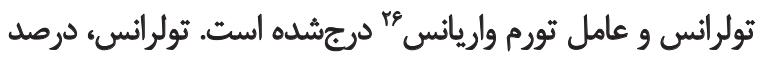

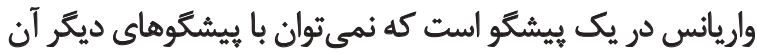

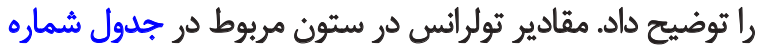

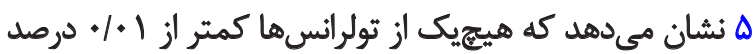

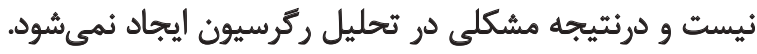

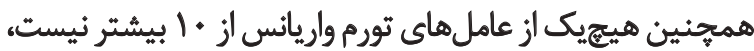

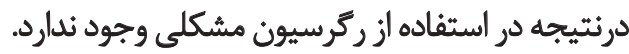

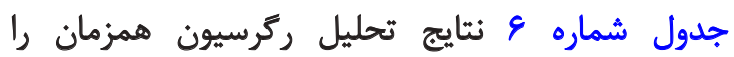

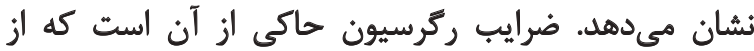

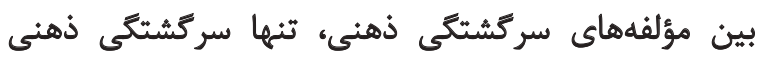

24. Durbin- Watson

25. Multicolinearity

26. Variance Inflation Factor (VIF)
يافتهها

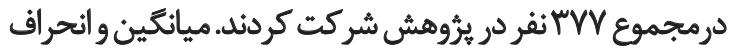

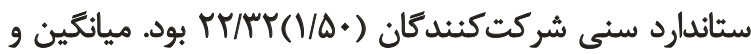

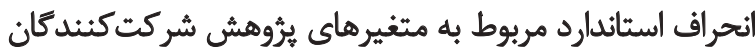

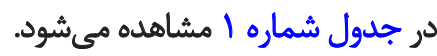

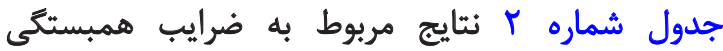

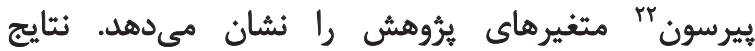

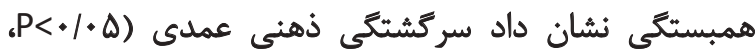

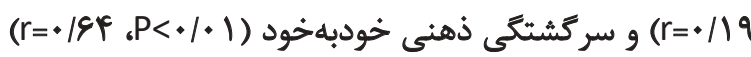
با نشائهاي وسواس رابطه مثيت و و مستقيم داردئ داردئ بدين

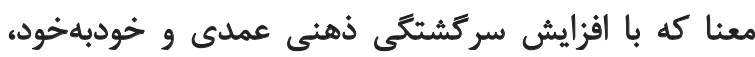

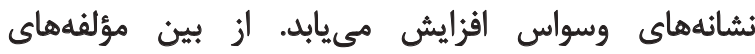

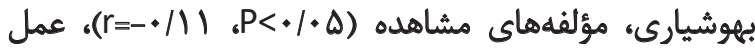

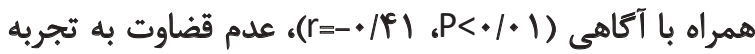

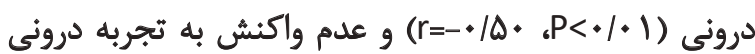

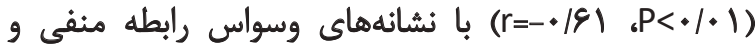

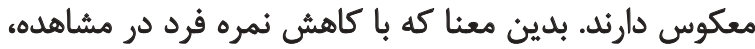

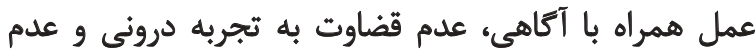

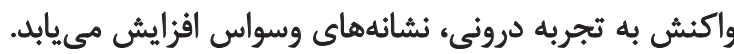

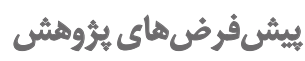

\section{ي ييش فرض طبيعى بودن دادهها}

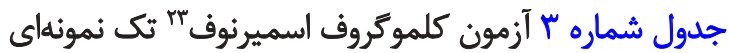

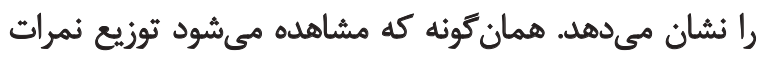
متغيرهاى يرؤهش با هو درصد اطمينان طبيعى است.

22. Pearson Correlation Coefficient

23. Kolmogorov-Smirnov

\begin{tabular}{|c|c|}
\hline ميانكين (انحراف استاندارد) & متغير \\
\hline$\Delta 1 / 4+(1+/ 4 \lambda)$ & نشانهماي وسواس \\
\hline$I F / N \Delta(F / \Lambda \Delta)$ & سركشتكي ذهنى عملى \\
\hline$r \cdot M q\left(1 F / M^{*}\right)$ & سركُشتكى ذهنى خودبلهُود \\
\hline$M \psi / \mu+(r / q V)$ & 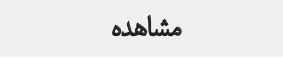 \\
\hline$r \cdot / \Delta F\left(\Delta / \mu^{\mu}\right)$ & توصيف \\
\hline TNAI(Y/AF) & عمل همراه با آكاهى \\
\hline$r / M I(r / M r)$ & عدم قضاوت به تجربه درونى \\
\hline IV/TV(Y/Tq) & عدم واكنش به تجربه دروتى \\
\hline
\end{tabular}

جدول ا. آمار توصيفى مربوط به متغيرهاي يُروهش 
جدول r. ماتريس همبستكى سركشتكى ذهنى عمدى و خودبهخود و بهوشيارى زمينهاي با نشانهاي وسواس

\begin{tabular}{|c|c|c|c|c|c|c|c|c|c|}
\hline \multirow[t]{8}{*}{$\Lambda$} & $Y$ & 7 & $\bullet$ & $\varepsilon$ & $r$ & r & 1 & مثغير & \\
\hline & & & & & & & 1 & نشانههاى وسواس & 1 \\
\hline & & & & & & 1 & $.119^{\circ}$ & سر كشتئكى ذهنى عملى & r \\
\hline & & & & & 1 &.$/ 49 * *$ & +194en & سركُشتكي ذهنينى & r \\
\hline & & & & 1 &.$/ 1 r * *$ &.$/ 11 * *$ & $-+111^{*}$ & مشاهده & $p$ \\
\hline & & & 1 & $\cdot \mid \Delta r^{* * *}$ & $-\cdot / 1 r$ & $\cdot / \pi$ & -+10 & توصيف & $\Delta$ \\
\hline & & 1 & . &.$/ \Lambda^{* * *}$ &.$- / M I^{* *}$ & $-* / 199^{*}$ &.$-|r| * \infty$ & عمل همراه با آكاهى & 8 \\
\hline & 1 &.$/ r q * *$ & $. / P)^{* * *}$ & $\cdot / p+*$ & $-+\mid(t) * *$ & $-* / M Y^{* *}$ &.$- / \Delta_{*}^{* *}$ & عدم قضضاوت به تجربه & $\checkmark$ \\
\hline 1 & . RE"** &.$/ M r * *$ & $.10 * *$ & $\cdot / 4+*$ &.$- / P V^{* *}$ &.$\pi$ &.$-|8|^{* * *}$ & عدم واكنش به تجربه & 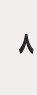 \\
\hline
\end{tabular}

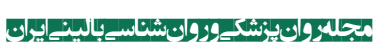

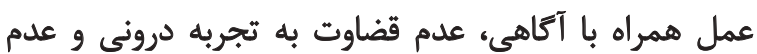

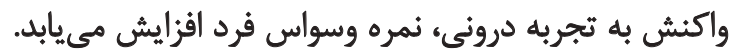
جدول شماره V نتايج تحليل واريانس را براي معناداري مدل

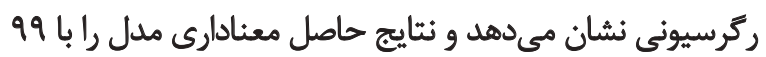

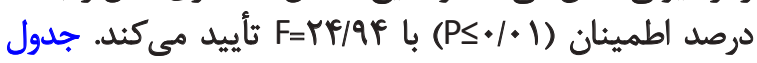

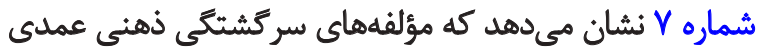

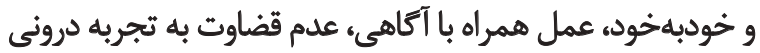

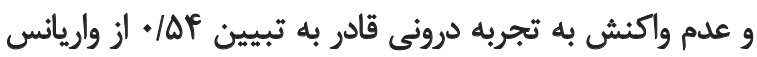

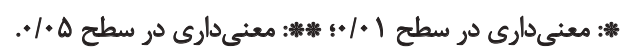

خودبلخود قادر به بيشبيشينى نشانههاي وسواس است. ضريب

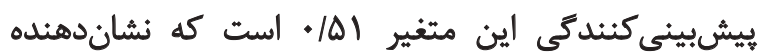

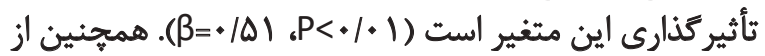

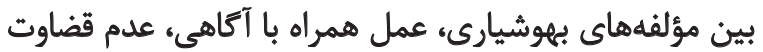

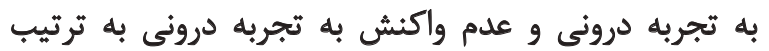

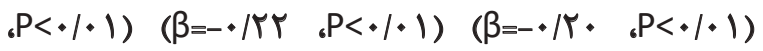

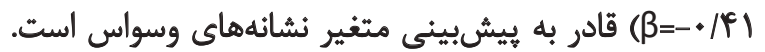

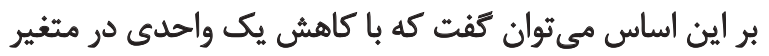

جدول r. آزمون كلموكراف اسميرنف براى بررسي نرمال بودن مثغيرهاى برُوهُش

\begin{tabular}{|c|c|c|}
\hline سطح معنى داري & Zo, أlo & متغير \\
\hline.$/ 918$ & . IOMf & سركشئكي ذَهني عمدى \\
\hline . lart & $\cdot / A \cdot P$ & سر كششكى ذهنى خودبلهخود \\
\hline.$/ 01 P$ & .1119 & مشاهلده \\
\hline VAV & . & توصيف \\
\hline$\cdot(T A)$ &.$/ 99$. & عمل همراه با آكاهي \\
\hline$\cdot|r \Delta|$ & | & عدم قضاوت به تجربه درونى \\
\hline.$/ 911$ & $\cdot|\Delta \infty|$ & عدم واكنش به تجربه درونى \\
\hline
\end{tabular}

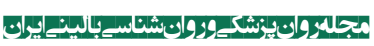

جدول ₹. نتايج آزمون دوربين واتسون براي استقلال مشاهدات

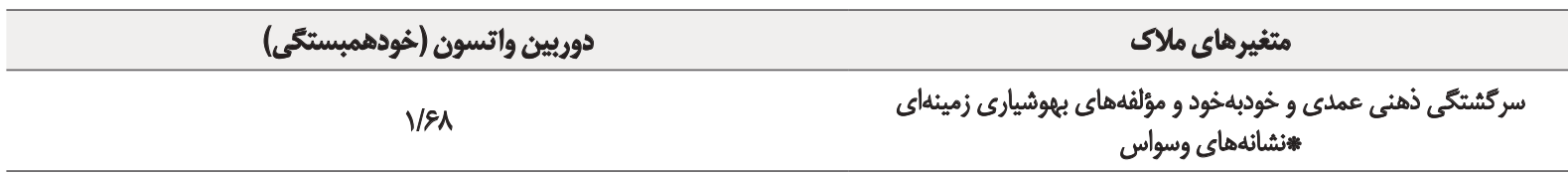


جدول ه. ضريب تولرانس و تورم واريانس متغيرهاى ثروهش

\begin{tabular}{|c|c|c|}
\hline VIF & تولرانس & مثغير \\
\hline$v / r q$ & $\cdot M$ & سركشتكى ذهنى عمدى \\
\hline $1 / \cdot 1$ & /AH & سركشتكى ذهنى خودبهنود \\
\hline $1 / \pi$ &.$/ V E$ & مشاهله \\
\hline V/Ai & . Ma & توصيف \\
\hline $1 / \cdot 9$ & .191 & عمل همراه با أكاهي \\
\hline $1 / 4$ & $\cdot / \Delta P^{\circ}$ & عدم قضاوت به تجربه دروئى \\
\hline $1 / 19$ & $\cdot M r$ & عدم واكنش به تجربه درونى \\
\hline
\end{tabular}

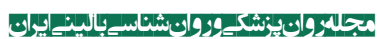

در درمان وسواس هم مؤثر باشد. فهميدن مكانيزمهاى زيربنايى متغير نشانههاى وسواس بودئد.

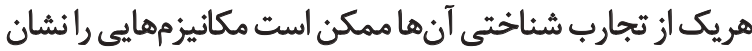

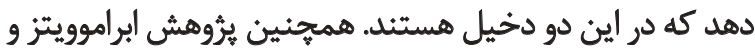

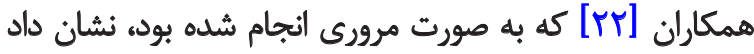

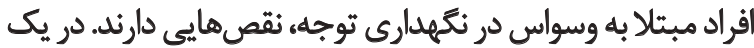

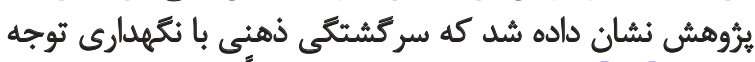

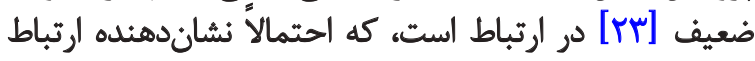
مكانيزمهاى زيربنايى دو مؤلفه است.

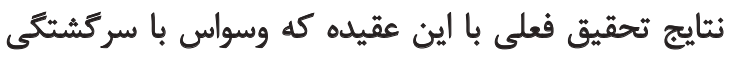

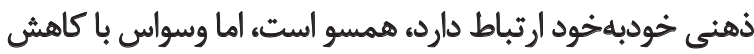

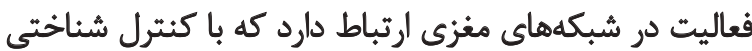

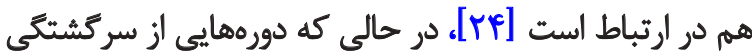

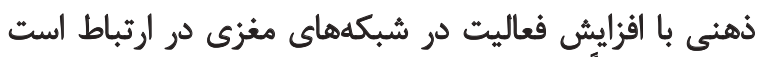

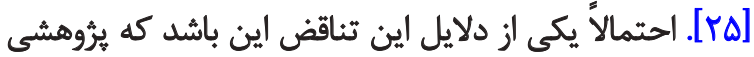

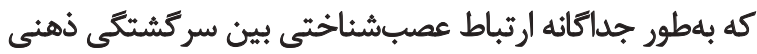

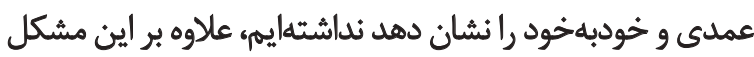

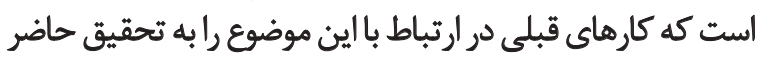

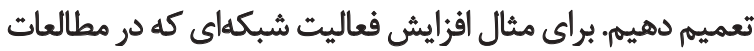

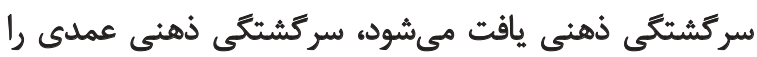

$$
\text { بعث }
$$

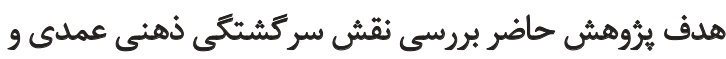

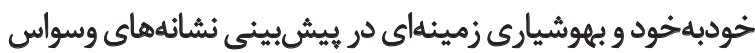

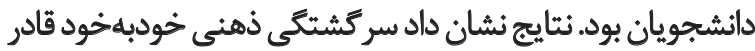
به بيشبينى نشانهانى وسواس دانشجويان است.

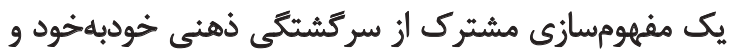
فكر وسواسى اين است كه زمانى اتفاق مي افتثد كه افر افراد، شكست

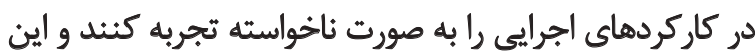

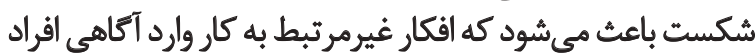

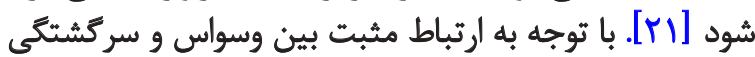

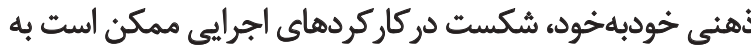

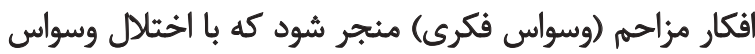

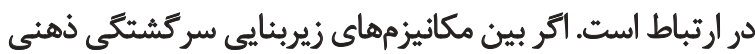

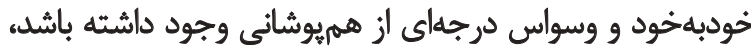

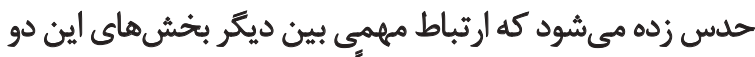

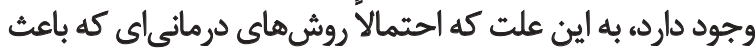

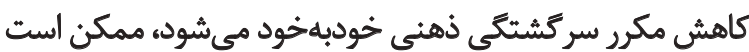

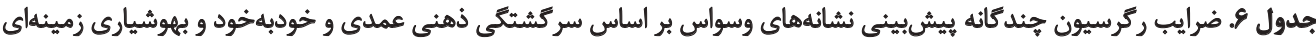

\begin{tabular}{|c|c|c|c|c|c|}
\hline Sig. & $\mathbf{T}$ & B & SE $\beta$ & $\mathbf{B}$ & متغير \\
\hline.$/ \Delta H$ &.$/ 8 Y$ & -.1 .4 & .1 .9 & -.1 .8 & سر كُشكي ذهنى عمدى \\
\hline $.1 . .1$ & $|V / D|$ & $\cdot|\Delta|$ & $.1 \cdot r$ &.$/ 4$ & سركشتكى ذهنى خودبيهُود \\
\hline . MP. & $-1 / 48$ & $-.1+\Delta$ &.$/ 11$ &.$- / 1 \mathrm{r}$ & هشاهله \\
\hline .1 .8$. & $V / M$ & .1 .8 & $\cdot / \pi r$ & .1 .8 & توصيف \\
\hline $.1 .+1$ & te/re & $-* / 4$ &.$/ \mathrm{r}$ & -+10 & عمل همراه با آكاهى \\
\hline .1 .14 & $-Y / 99$ &.$- / M r$ &.$/ 11$ &.$- / M \varphi$ & عدم قضاوت به تجربه درونى \\
\hline $.1 . .1$ & $-.9 / n^{e}$ &.$- \mid p i$ &.$/ 19$ & $-1 / A C$ & عدم واكنش به تجربه درونى \\
\hline
\end{tabular}


جدول V. خلاصه مدل ركرسيون، تحليل واريانس و مشخصههاى آمارى ركرسيون نشانهاي وسواس از روى متغيرهاى بيشبين

\begin{tabular}{|c|c|c|c|c|c|c|c|}
\hline سطح معنى دارى & $\mathbf{F}$ & $\mathbf{R}^{2}$ & Adjusted $\mathbf{R}^{2}$ & مياتكينين مجذورات & df & مجموع مجذورات & منابع \\
\hline \multirow[t]{3}{*}{$+1+\infty 1$} & TF/qY & $\cdot / \Delta \Delta$ & . IAF & 1099.110 & $v$ & MTEYE/.0 & ركرسيون \\
\hline & & & & $\mathrm{rV} / \mathrm{Q}$ & HAT & $1059 . / 19$ & باقيىمانده \\
\hline & & & & & พq. & PVEYIED & كل \\
\hline
\end{tabular}

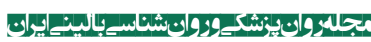

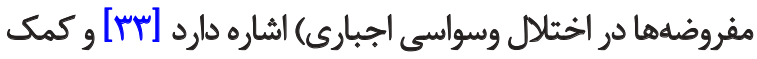

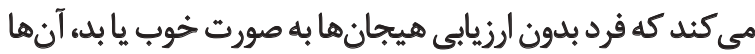

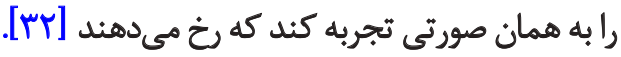

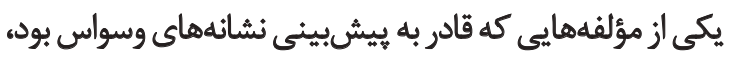

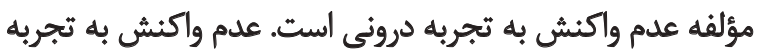

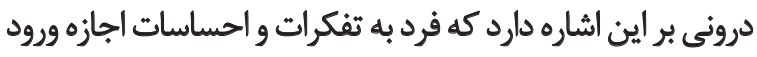

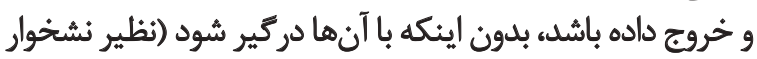

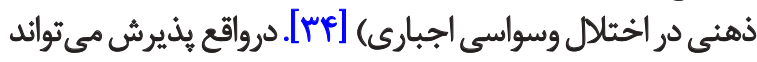

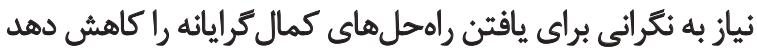

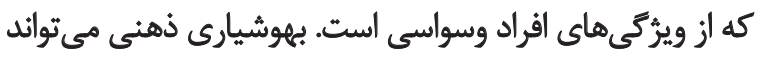

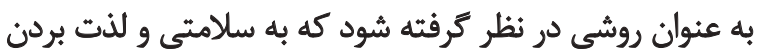

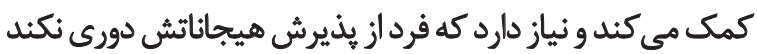

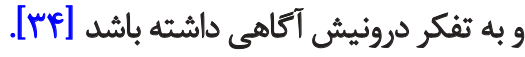

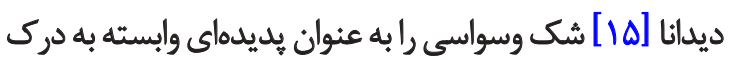

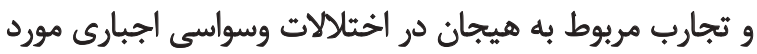

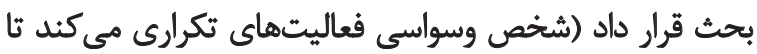

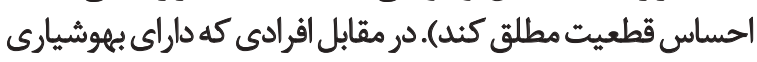

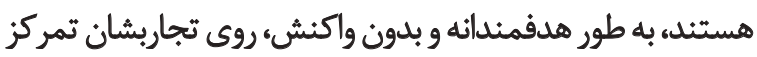

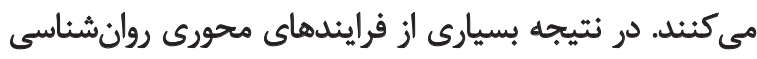

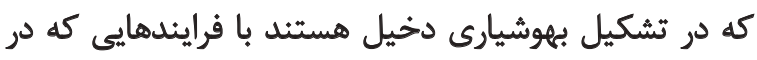
اختلالات وسواس اجبارى دخيل هستند، تضاد دارند.

نتيجهئيرى

يافتهها نشان داد متغير سركشتكى ذهنى غيرعمدى قادر به به يه

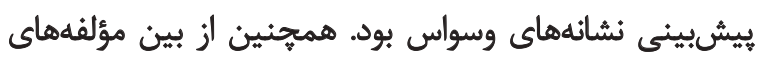

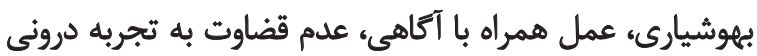

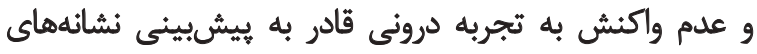

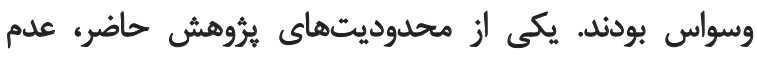

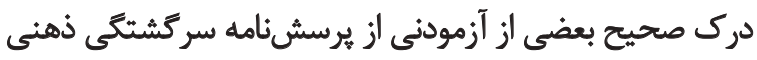

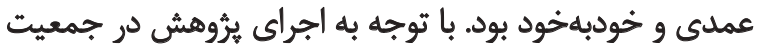

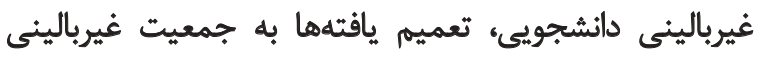

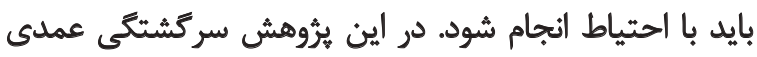

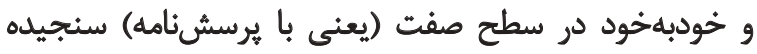

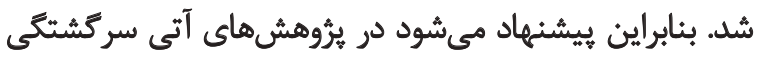

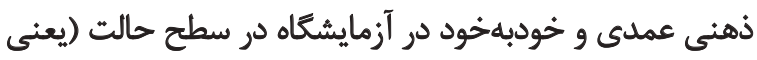

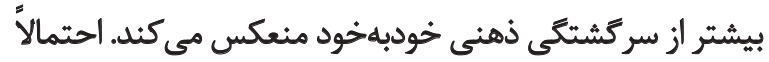

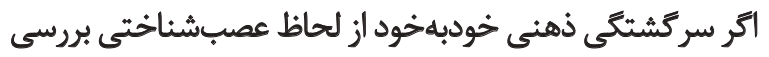
شود، شايد كاهش فعاليت در شبكهنهاى مغزى رانه را نشان دهد.

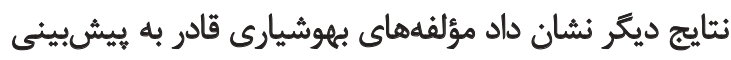
نشانههاي وسواس بودند. اين يافته با يافتهانهاى بابإيور و همكاراران

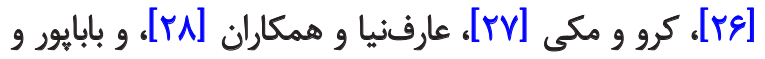

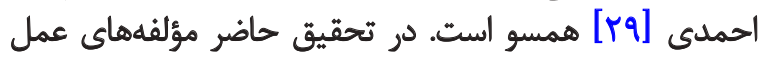

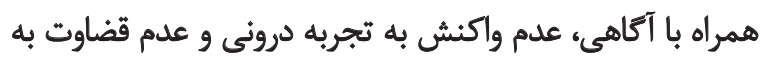

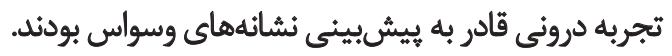
يكى از جنبههاي بهوشيارى زمينهاي، عمل همراه با آكاهى

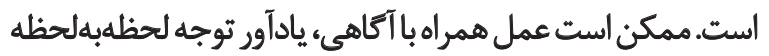

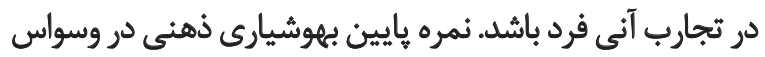

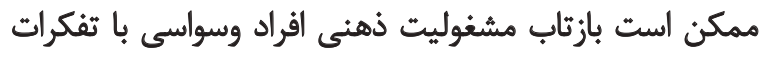

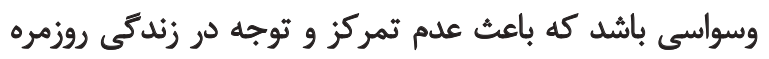

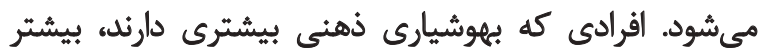

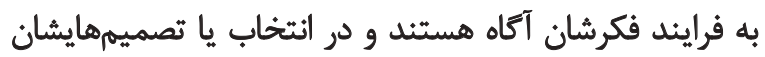

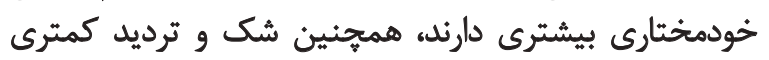
نسبت امور دارند.

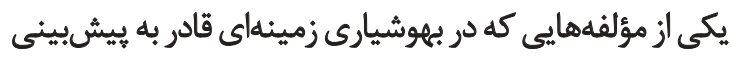

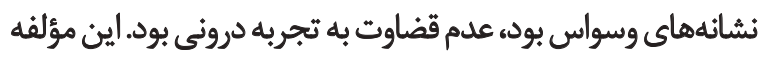

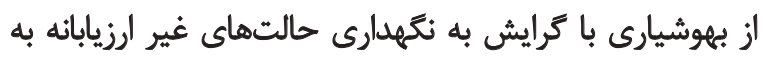

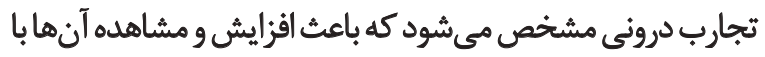

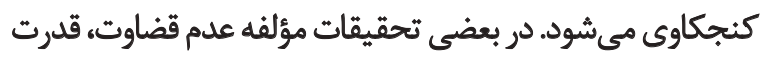

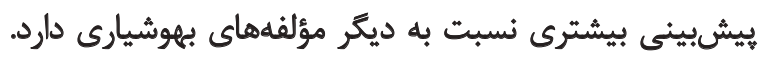

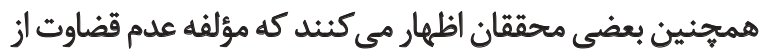

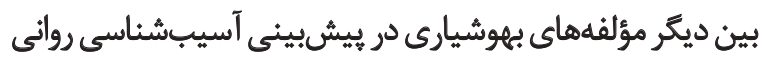

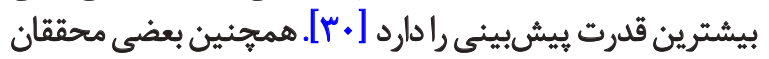

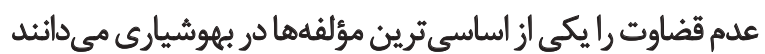

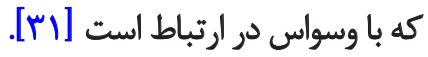

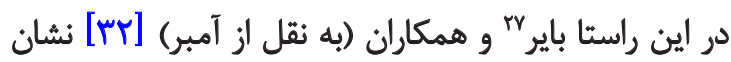

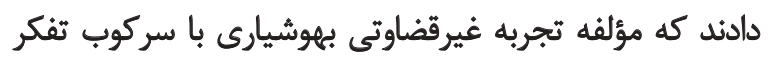

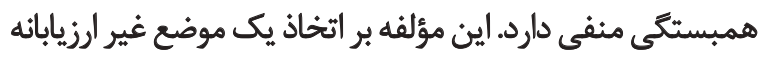

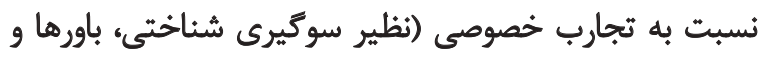


با استفاده از ابزارهاى آزمايشى) در افراد مبتلا به وسواس فكرى

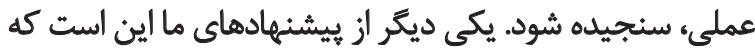

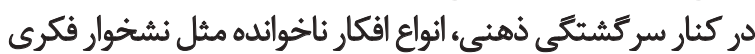

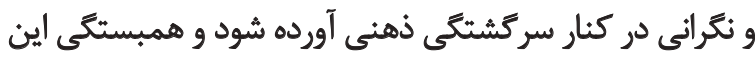

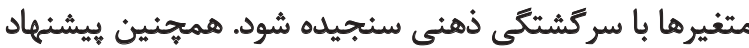

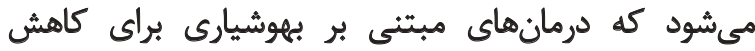

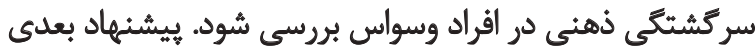

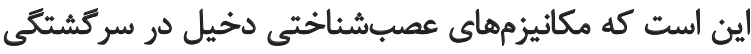
ذهنى غيرعمدى و عمدى بررسى شود.

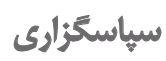

اين مقاله بركرفته از بايان نامه كارشناسى ارشد آثاى بهرئ

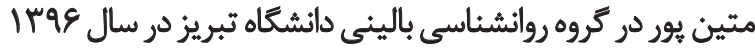

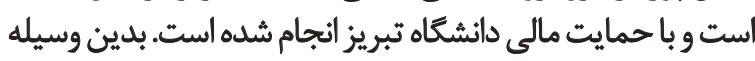

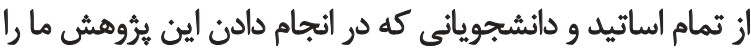

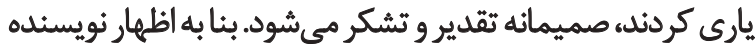
مسئول مقاله، تعارض منافع وجود نداشته است. 


\section{References}

[1] American Psychiatric Association. Diagnostic and statistical manual of mental disorders (DSM-5®). Washington, D.C.: American Psychiatric Pub; 2013. [DOI:10.1176/appi.books.9780890425596]

[2] Nelson JD. Mental pollution and inflated responsibility in obsessive-compulsive disorder: The contribution of anxiety, disgust, and guilt (PhD dissertation). Bronx: Fordham University; 2005.

[3] Verkuil B, Brosschot JF, Thayer JF. Capturing worry in daily life: Are trait questionnaires sufficient. Behaviour Research and Therapy. 2007; 45(8):1835-44. [DOI:10.1016/j.brat.2007.02.004] [PMID]

[4] Christoff K, Gordon A, Smith R, Vancouver BC. The role of spontaneous thought in human cognition. In Vartanian O, Mandel DR, editors. Neuroscience of decision making. New York: Psychology Press; 2011.

[5] Smallwood J, Schooler JW. The restless mind. Psychological Bulletin. 2002; 132(6):946. [DOI:10.1037/0033-2909.132.6.946] [PMID]

[6] Schooler JW. Re-representing consciousness: Dissociations between experience and meta-consciousness. Trends in Cognitive Sciences. 2002; 6(8):339-44. [DOI:10.1016/S1364-6613(02)01949-6]

[7] Seli P, Carriere JS, Smilek D. Not all mind wandering is created equal: Dissociating deliberate from spontaneous mind wandering. Psychological Research. 2015; 79(5):750-8. [DOI:10.1007/ s00426-014-0617-x] [PMID]

[8] Seli P, Risko EF, Purdon C, Smilek D. Intrusive thoughts: Linking spontaneous mind wandering and OCD symptomatology. Psychological Research. 2017; 81(2):392-8. [DOI:10.1007/s00426016-0756-3] [PMID]

[9] Carriere JS, Seli P, Smilek D. Wandering in both mind and body: Individual differences in mind wandering and inattention predict fidgeting. Canadian Journal of Experimental Psychology/ Revue canadienne de psychologie expérimentale. 2013; 67(1):19. [DOI:10.1037/a0031438] [PMID]

[10] Kane MJ, Brown LH, McVay JC, Silvia PJ, Myin-Germeys I, Kwapil TR. For whom the mind wanders, and when: An experience-sampling study of working memory and executive control in daily life. Psychological Science. 2007; 18(7):614-21. [DOI:10.1111/j.1467-9280.2007.01948.x] [PMID]

[11] Killingsworth MA, Gilbert DT. A Wandering mind is an unhappy mind. Science. 2010; 330(6006):932-932. [DOI:10.1126/scii ence.1192439] [PMID]

[12] Smith JM, Alloy LB. A roadmap to rumination: A review of the definition, assessment, and conceptualization of this multifaceted construct. Clinical Psychology Review. 2009; 29(2):116-28. [DOI:10.1016/j.cpr.2008.10.003] [PMID] [PMCID]

[13] Williams JMG. Mindfulness, depression and modes of mind. Cognitive Therapy and Research. 2008; 32(6):721. [DOI:10.1007/ s10608-008-9204-z]

[14] Salkovskis PM. Cognitive-behavioural factors and the persistence of intrusive thoughts in obsessional problems. Behaviour Research and Therapy. 1989; 27(6):677-82. [DOI:10.1016/00057967(89)90152-6]

[15] Didonna F. Mindfulness and obsessive-compulsive disorder: Developing a way to trust and validate one's internal experience. Berlin: Clinical Handbook of Mindfulness: Springer; 2009.
[16] Esfand Zad AH, Shams G, Meysami AP, Erfan A. [The role of mindfulness, emotion regulation, distress tolerance and interpersonal effectiveness in predicting obsessive-compulsive symptoms (Persian)]. Iranian Journal of Psychiatry \& Clinical Psychology. 2017; 22(4):270-83.

[17] Hasker SM. Evaluation of the mindfulness-acceptance-commitment (MAC) approach for enhancing athletic performance (PhD dissertation). Indiana, Pennsylvania: Indiana University of Pennsylvania; 2010.

[18] Heydarinasab L. [An investigation of the validity and reliability of psychometric characteristics of five facet mindfulness questionnaire in Iranian non-clinical samples(Persian)]. International Journal of Behavioral Sciences. 2013; 7(3):229-37.

[19] Rachman SJ, Hodgson RJ. Obsessions and compulsions. New Jersey: Prentice Hall; 1980.

[20] Zahed A, Ghalilo K, Abolghasemi A, Narimani M. The relationship between emotion regulation strategies and interpersonal behavior among substance abusers. Research on Addiction. 2009; 3(11):99-114.

[21] KaneMJ,McVayJC. What mind wandering reveals aboutexecutive-control abilities and failures. Current Directions in Psychological Science. 2012; 21(5):348-54. [DOI:10.1177/0963721412454875]

[22] Abramovitch A, Cooperman A. The cognitive neuropsychology of obsessive-compulsive disorder: A critical review. Journal of Obsessive-Compulsive and Related Disorders. 2015; 5:24-36. [DOI:10.1016/j.jocrd.2015.01.002]

[23] Seli P, Cheyne JA, Smilek D. Wandering minds and wavering rhythms: Linking mind wandering and behavioral variability. Journal of Experimental Psychology: Human Perception and Performance. 2013; 39(1):1. [DOI:10.1037/a0030954]

[24] Koçak OM, Özpolat AY, Atbaşoğlu C, Çiçek M. Cognitive control of a simple mental image in patients with obsessivecompulsive disorder. Brain and Cognition. 2011; 76(3):390-9. [DOI:10.1016/j.bandc.2011.03.020] [PMID]

[25] Christoff K, Gordon AM, Smallwood J, Smith R, Schooler JW. Experience sampling during fMRI reveals default network and executive system contributions to mind wandering. Proceedings of the National Academy of Sciences. 2009; 106(21):8719-24. [DOI:10.1073/pnas.0900234106] [PMID] [PMCID]

[26] Babapour Kaj, Poursharifi H, Hashemi T, Ahmadi E. The relationship of meta-cognition and mindfulness components with obsessive beliefs in students. Journal of School Psychology. 2013; 1(4);23-38.

[27] Crowe K, McKay D. Mindfulness, obsessive-compulsive symptoms, and executive dysfunction. Cognitive Therapy and Research. 2016; 40(5):627-44. [DOI:10.1007/s10608-016-9777-x]

[28] Arefnia R, Bagheri M. [The Relationship between Mindfulness and Perfectionism with Obsessive Thoughts in Psychology Students of Islamic Azad University of Karaj (Persian)]. Paper presented at the National Conference on Psychology and Social Injury Management. 9 March 2016; Chabahar, Iran.

[29] Babapoor J, Ahmadi E. [A comparison of emotion regulation strategies and mindfulness in students with and without obsessive believes (Persian)]. Modern Psychological Research. 2013; 7(28):30-47

[30] Cash M, Whittingham K. What facets of mindfulness contribute to psychological well-being and depressive, anxious, and 
stress-related symptomatology. Mindfulness. 2010; 1(3):177-82. [DOI:10.1007/s12671-010-0023-4]

[31] Moritz S, Wess N, Treszl A, Jelinek L. The attention training technique as an attempt to decrease intrusive thoughts in $\mathrm{Ob}$ sessive-Compulsive Disorder (OCD): From cognitive theory to practice and back. Journal of Contemporary Psychotherapy. 2011; 41(3):135-43. [DOI:10.1007/s10879-010-9169-6]

[32] Emanuel AS. The role of mindfulness in affective forecasting (PhD dissertation). Ohio: Kent State University; 2009.

[33] Kabat Zinn J. Mindfulness based interventions in context: Past, present, and future. Clinical Psychology: Science and Practice. 2003; 10(2):144-56. [DOI:10.1093/clipsy.bpg016]

[34] Baer RA. Mindfulness training as a clinical intervention: A conceptual and empirical review. Clinical Psychology: Science and Practice. 2003; 10(2):125-43. [DOI:10.1093/clipsy.bpg015] 
